\title{
Convergence of SAOR Method for the Linear Complementarity Problems ${ }^{1}$
}

\author{
${ }^{\mathrm{a}}$ Xian-li Han, ${ }^{\mathrm{b}}$ Dong-jin Yuan, ${ }^{\mathrm{c}}$ Shan Jiang \\ College of Mathematics Science, Yangzhou University, Yangzhou, Jiangsu Province, China
}

\begin{abstract}
In this paper we apply an iterative method, the SAOR method for solving the linear complementarity problem, and some sufficient conditions for the convergence of the new method are presented when the system matrix $\mathrm{M}$ is an M-matrix. Moreover when M is an L-matrix, we discuss the monotone convergence of it. Finally, we report the numerical results of our proposed method.
\end{abstract}

Index Terms: Linear complementarity problem; SAOR method; Convergence; H-matrix; M-matrix

(C) 2012 Published by MECS Publisher. Selection and/or peer review under responsibility of the Research Association of Modern Education and Computer Science.

\section{Introduction}

For a given matrix $M$ and a vector $q$, the linear complementarity problem (LCP) consists of finding a vector $z$ which satisfies the conditions

$$
z \geq 0, M z+q \geq 0, z^{T}(M z+q)=0
$$

Since there is a variety of applications such as Nash equilibrium point of a matrix game, the contact problems and the free boundary problem for bearings, etc..(see [1]) The researches on the numerical methods for solving (1) have been attracted much attention.

A large number of papers have studied the LCP. In [2], several basic iterative methods to solve LCP are discussed. Recently, some new iterative methods have been proposed to solve LCP. For example, Koulisianis and Papatheodorou [3] present an improved projected successive overrelaxation (IPSOR) for the solution of an

\footnotetext{
${ }^{1}$ This work is supported by NSF Grant \#11026113 to S. Jiang.

* Corresponding author.

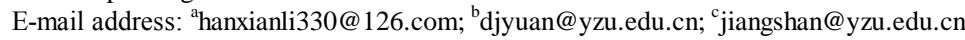


important class of linear complementarity problems. When $\mathrm{M}$ is a 2-cyclic matrix, Yuan and Song [4] propose a class of modified AOR (MAOR) method to solve it. In [5], a class of generalized AOR (GAOR) methods are introduced. And by applying the multisplitting techniques, Bai [6,7] propose a class of parallel iterative methods for a large linear complementarity problem.

In this paper, by applying the splitting we propose the SAOR method for solving the linear complementarity problem. Convergence results for the method are presented when $\mathrm{M}$ is an H-matrix (and also an M-matrix). Finally, a numerical example is given to show the efficiency of our presented method.

Definition 1.1. Let $A=\left(a_{i, j}\right) \in R^{n \times n}$, and it is called

1) L-matrix if $a_{k k}>0, k=1,2, \ldots, n$, and $a_{k j} \leq 0, k \neq j, k, j=1,2, \ldots, n$;

2) M-matrix if it is a nonsingular L-matrix satisfying $A^{-1} \geq 0$;

3) S- matrix if $\exists x>0, A x>0$;

4) H-matrix if $\langle A\rangle$ is an M-matrix;

5) $H_{+}$-matrix if A is an H-matrix and $a_{k k}>0, k=1,2, \ldots, n$.

Definition 1.2. For $A=\left(a_{i, j}\right) \in R^{n \times n}$, vector $x_{+}$is defined such that $\left(x_{+}\right)_{j}=\max \left\{0, x_{j}\right\}, j=1,2, \ldots, n$. Then for any $x, y \in R^{n}$, the following facts hold: [6]

1) $(x+y)_{+} \leq x_{+}+y_{+}$

2) $x_{+}-y_{+} \leq(x-y)_{+}$;

3) $|x|=x_{+}+(-x)_{+}$;

4) $x \leq y \Rightarrow x_{+} \leq y_{+}$

Lemma 1.1. [8] Let $A=M-N$ be an M-splitting, then $\rho\left(M^{-1} N\right)<1 \Leftrightarrow A$ is a nonsingular M-matrix.

Lemma 1.2. [7] Let $\mathrm{M}$ is an $H_{+}$-matrix, then $\operatorname{LCP}(\mathrm{M}, \mathrm{q})$ has an unique solution $z \in R^{n}$.

Lemma 1.3. [1] $A$ is an M-matrix if and only if $A$ is a $Z$ - matrix and an S-matrix.

Lemma 1.4. [9] Let $A_{1}$ is an M-matrix and

$D_{1}=\operatorname{diag}\left(A_{1}\right), C_{1}=D_{1}-A_{1}$. If $D_{2} \geq 0 \in R^{n \times n}$ is a diagonally matrix and $C_{2} \in R^{n \times n}$ with $0 \leq C_{2} \leq C_{1}$, then $A=\left(D_{1}-D_{2}\right)-\left(C_{1}-C_{2}\right)$ and $A^{-1} \leq A_{1}^{-1}$. 


\section{SAOR Method For LCP(M,q)}

Let $M=D-B=D-L-U$, where $D=\operatorname{diag}(M)=\left(d_{i j}\right), L=\left(l_{i j}\right)$, and $U=\left(u_{i j}\right)$ are diagonal, strictly lower and upper triangular matrices obtained from $\mathrm{M}$, respectively. It has been shown in $[10,11]$ that $z^{*}$ solves $\mathrm{LCP}(\mathrm{M}, \mathrm{q})$ (1) if and only if it satisfies $Z^{*}=\left(Z^{*}-D(M Z+q)\right)_{+}$where $D=\operatorname{diag}(M)$.

Now we propose the new iterative SAOR method for solving LCP(M,q) (1) as follows.

\section{SAOR method for LCP}

Step 1: Choose an initial vector $z^{0} \in R^{n}$ and a parameter $\omega, \gamma \in R^{+}$, set $k=0$;

Step 2: Calculate

$$
\begin{aligned}
z^{k+1}= & \left(z^{k}-D^{-1}\left\{-\gamma L z^{k+1}+[\omega(2-\omega) M+\gamma L] z^{k}\right.\right. \\
& +\omega(2-\omega) q\})_{+}
\end{aligned}
$$

Step 3: If $z^{k+1}=z^{k}$, then stop; Else if, set $k=k+1$ and go to Step 2 .

\section{Remark.}

1) if $\omega=\gamma$, then SAOR method reduces to SSOR method;

2) if $\omega=1$, then SAOR method reduces to AOR method;

3) if $\omega=\gamma=1$, then SAOR method reduces to SOR method.

\section{Convergence Analysis For $H_{+}$-Matrix}

At first we define the operator $f: R^{n} \rightarrow R^{n}$ in accordance with the rule: $f(z)=\xi$, where $\xi$ is the fixed point of the system of equations

$$
\xi=\left(z-D^{-1}\{-\gamma L \xi+[\omega(2-\omega) M+\gamma L] z+\omega(2-\omega) q\}\right)_{+}
$$

we can prove the following convergence theorem for the SAOR method.

Theorem 3.1. Let $M=D-B=D-L-U$ be an $H_{+}$-matrix and $D=\operatorname{diag}(M), L, U$ be diagonal, strictly lower and upper triangular matrices obtained from $\mathrm{M}$, respectively. If $0<\gamma \leq \omega \leq 1$, then for any initial vector $z^{0}$, the SAOR method converge to the unique solution of $\operatorname{LCP}(M, q)$.

Proof. By Lemma 1.2, LCP(M,q) has an unique solution $z^{*} \in R^{n}$, that is $f\left(z^{*}\right)=z^{*}$. Suppose that $\eta=f(y)$, i.e.

$$
\eta=\left(y-D^{-1}\{-\gamma L \eta+[\omega(2-\omega) M+\gamma L] y+\omega(2-\omega) q\}\right)_{+}
$$

then by subtracting (3) from (2), we get 


$$
\begin{aligned}
\xi-\eta & =\left(z-D^{-1}\{-\gamma L \xi+[\omega(2-\omega) M+\gamma L] z+\omega(2-\omega) q\}\right)_{+} \\
& -\left(y-D^{-1}\{-\gamma L \eta+[\omega(2-\omega) M+\gamma L] y+\omega(2-\omega) q\}\right)_{+} \\
& \leq\left((z-y)-D^{-1}\{-\gamma L \xi+[\omega(2-\omega) M+\gamma L](z-y)\}\right)_{+} \\
& =\left(\gamma D^{-1} L(\xi-\eta)+\left(\left\{I-D^{-1}[\omega(2-\omega) M+\gamma L]\right\}(z-y)\right)\right)_{+},
\end{aligned}
$$

So

$$
\begin{aligned}
(\xi-\eta)_{+} \leq & \left(\gamma D^{-1} L(\xi-\eta)\right)_{+} \\
& +\left\{\left(I-D^{-1}[\omega(2-\omega) M+\gamma L]\right)(z-y)\right\}_{+}
\end{aligned}
$$

Analogously, we can obtain

$$
(\eta-\xi)_{+} \leq\left(\gamma D^{-1} L(\eta-\xi)\right)_{+}+\left(\left(I-D^{-1}[\omega(2-\omega) M+\gamma L]\right)(y-z)\right)_{+} .
$$

Now, the combination of (4) and (5) gives the following estimates

$$
\begin{aligned}
& |\xi-\eta|=(\xi-\eta)_{+}+(\eta-\xi)_{+} \\
& \leq\left(\gamma D^{-1} L(\xi-\eta)\right)_{+}+\left(\gamma D^{-1} L(\eta-\xi)\right)_{+} \quad+\left(\left(I-D^{-1}[\omega(2-\omega) M+\gamma L]\right)(z-y)\right)_{+} \\
& +\left(\left(I-D^{-1}[\omega(2-\omega) M+\gamma L]\right)(y-z)\right)_{+} \\
& =\left|\gamma D^{-1} L(\xi-\eta)\right|+\left|\left(I-D^{-1}[\omega(2-\omega) M+\gamma L]\right)(z-y)\right| \leq \gamma D^{-1}|L||(\xi-\eta)|+\left|\left(I-D^{-1}[\omega(2-\omega) M+\gamma L]\right)\right||z-y| \\
& \text { i.e., }\left(I-\gamma D^{-1}|L|\right)|\xi-\eta| \leq\left|I-D^{-1}[\omega(2-\omega) M+\gamma L]\right| z-y \mid \text {, } \\
& \text { let } Q=I-\gamma D^{-1}|L|, R=\mid I-D^{-1}[\omega(2-\omega) M+\gamma L] \text {, }
\end{aligned}
$$

then 


$$
|\xi-\eta| \leq Q^{-1} R|z-y|
$$

According to the SAOR method and (6), we can get

$$
\left|z^{k+1}-z^{*}\right|=\left|f\left(z^{k}-z^{*}\right)\right| \leq Q^{-1} R\left|z^{k}-z^{*}\right|
$$

by Lemma 1.2 it is easy to see that the iterative sequence $\left\{z^{k}\right\}, k=0,1,2, \ldots$ generated by the SAOR method converges to the unique solution $z^{*}$ if $\rho\left(M^{-1} N\right)<1$.

Corollary 3.2. Let $M=D-B=D-L-U$ be an M-matrix and $D=\operatorname{diag}(M), L, U$ be diagonal, strictly lower and upper triangular matrices obtained from $\mathrm{M}$, respectively. If $0<\gamma \leq \omega \leq 2$, then for any initial vector $z^{\circ}$, the SAOR method converge to the unique solution of LCP(M,q).

\section{Monotone Convergence Analysis}

In this section, we discuss the monotone convergence properties of the SAOR method when the system matrix $\mathrm{M} \in \mathrm{R}^{n \times n}$ is an L-matrix. And we define the following set $\Delta=\left\{x \in R^{n} \mid x \geq 0, M x+q \geq 0\right\}$.

Theorem 4.1. Let the operator $f: R^{n} \rightarrow R^{n}$ be defined in (2). Suppose that $\mathrm{M} \in \mathrm{R}^{n \times n}$ is an L-matrix, and $0<\gamma \leq \omega \leq 1$. Then for any $z \in \Delta$, it holds that
1) $f(z) \leq z$
2) $y \leq z \Rightarrow f(y) \leq f(z)$;
3) $\xi=f(z) \in \Delta$.

Proof. We firstly verify 1 ). To show that

$$
\xi_{i} \leq z_{i}, i=1,2, \ldots n
$$

where

$$
\xi_{i}=\left(z_{i}-d_{i i}^{-1}\left[-\gamma \sum_{j=1}^{i-1} L_{i j}\left(\xi_{j}-z_{j}\right)+\omega(2-\omega)(M z+q)_{i}\right]\right)_{+}
$$

We use induction on $\mathrm{i}$ to prove (7). When $\mathrm{i}=1$, noticing that $\mathrm{M}$ is an L-matrix, $0<\gamma \leq \omega \leq 1$ and $z \in \Delta$, $z_{1} \geq 0$ 


$$
d_{i i}^{-1}\left[\omega(2-\omega)(M z+q)_{i}\right] \geq 0
$$

thus

$$
\xi_{1}=\left(z_{1}-d_{i i}^{-1}\left[\omega(2-\omega)(M z+q)_{i}\right]\right)_{+} \leq z_{1}
$$

Now assume that (7) holds for $\forall i \leq k(0<k<n)$, then we get $z_{k+1} \geq 0$,

and

$$
d_{k+1 k+1}^{-1}\left[-\gamma \sum_{j=1}^{k} L_{i j}\left(\xi_{j}-z_{j}\right)+\omega(2-\omega)(M z+q)_{k+1}\right] \geq 0,
$$

thus

$$
\xi_{k+1}=\left(z_{k+1}-d_{k+1 k+1}{ }^{-1}\left[-\gamma \sum_{j=1}^{k} L_{i j}\left(\xi_{j}-z_{j}\right)+\omega(2-\omega)(M z+q)_{k+1}\right]\right)_{+} \leq z_{k+1},
$$

by induction, (7) holds for all $\mathrm{i}=1,2, \ldots, \mathrm{n}$.

To verify 2), we denote $\eta=f(y)$, where $\eta$ is the fixed point of the system of equation:

$$
\eta=\left(y-D^{-1}\{-\gamma L \eta+[\omega(2-\omega) M+\gamma L] y+\omega(2-\omega) q\}\right)_{+}
$$

Now we only need to show that

$$
\eta_{i} \leq \xi_{i} \text {, when } y \leq z, i=1,2, \ldots, n
$$

by noticing (8) we can obtain

$$
\begin{aligned}
\xi_{i} & =\left(z_{i}-d_{i i}^{-1}\left[-\gamma \sum_{j=1}^{i-1} L_{i j} \xi_{j}+\gamma \sum_{j=1}^{i-1} L_{i j} z_{j}+\omega(2-\omega) d_{i i} z_{i}\right.\right. \\
& -\omega(2-\omega) \sum_{j=1}^{i-1} L_{i j} z_{j}+\omega(2-\omega) \sum_{j=i+1}^{n} U_{i j} z_{j} \\
& \left.\left.+\omega(2-\omega)(M z+q)_{i}\right]\right)_{+}
\end{aligned}
$$




$$
\begin{aligned}
= & \left((\omega-1)^{2} z_{i}-d_{i i}^{-1}\left[-\gamma \sum_{j=1}^{i-1} L_{i j} \xi_{j}+\left(\omega^{2}-2 \omega+\gamma\right) \sum_{j=1}^{i-1} L_{i j} z_{j}\right.\right. \\
& \left.\left.-\omega(2-\omega) \sum_{j=i+1}^{n} U_{i j} z_{j}+\omega(2-\omega) q_{i}\right]\right)_{+},
\end{aligned}
$$

and

$$
\begin{aligned}
\eta_{i} & =\left((\omega-1)^{2} y_{i}-d_{i i}{ }^{-1}\left[-\gamma \sum_{j=1}^{i-1} L_{i j} \eta_{j}+\left(\omega^{2}-2 \omega+\gamma\right) \sum_{j=1}^{i-1} L_{i j} y_{j}\right.\right. \\
& \left.\left.-\omega(2-\omega) \sum_{j=i+1}^{n} U_{i j} y_{j}+\omega(2-\omega) q_{i}\right]\right)_{+} .
\end{aligned}
$$

Considering the hypotheses $y \leq z$, then when $i=1$, we obtain

$$
\begin{aligned}
\xi_{1} & =\left((\omega-1)^{2} z_{1}+d_{11}{ }^{-1}\left[\omega(2-\omega) \sum_{j=2}^{n} U_{1 j} z_{j}-\omega(2-\omega) q_{1}\right]\right)_{+} \\
& \geq\left((\omega-1)^{2} y_{1}+d_{11}^{-1}\left[\omega(2-\omega) \sum_{j=2}^{n} U_{1 j} y_{j}-\omega(2-\omega) q_{1}\right]\right)_{+}=\eta_{1}
\end{aligned}
$$

Now assume that (7) holds for $\forall i \leq k(0<k<n)$, when $i=k+1$, by (9)(10) and $y \leq z$ and hence $\eta_{k+1} \leq \xi_{k+1}$. Hence the conclusion 2) holds by the principle of induction.

The proof of 3) is omitted here.

Theorem 4.2. Let $\mathrm{M} \in \mathrm{R}^{n \times n}$ be an L-matrix, for any initial vector $z^{0}=y^{0} \in \Delta$, both the iterative sequences $\left\{z^{k}\right\}$ and $\left\{y^{k}\right\}$ generated by the SAOR method corresponding to the parameters $(\omega, \gamma)$ and $(\bar{\omega}, \bar{\gamma})$, respectively, converge to $z^{*}$ of $\mathrm{LCP}(\mathrm{M}, \mathrm{q})$ and holds

$$
z^{k} \leq y^{k}, k=0,1,2, \ldots
$$

provided parameters $(\omega, \gamma)$ and $(\bar{\omega}, \bar{\gamma})$ satisfy

$$
\begin{aligned}
& 0<\bar{\omega} \leq \omega \leq 1 ; 0<\bar{\gamma} \leq \gamma \leq 1 ; \\
& 0<\bar{\gamma} \leq \bar{\omega} \leq 1 ; 0 \leq \gamma \leq \omega \leq 1 .
\end{aligned}
$$

Proof. First we define the operator $f: R^{n} \rightarrow R^{n}$ such that $\xi=f(z)$, where $\xi$ is the fixed point of the following system 


$$
\begin{aligned}
& \xi=\left(z-D^{-1}\{-\gamma L \xi+[\omega(2-\omega) M+\gamma L] z+\omega(2-\omega) q\}\right)_{+} \text {Since } \\
& y^{p+1}=\left(y^{p}-D^{-1}\left\{-\bar{\gamma} L y^{p+1}+[\bar{\omega}(2-\bar{\omega}) M+\bar{\gamma} L] y^{p}+\bar{\omega}(2-\bar{\omega}) q\right\}\right)_{+}
\end{aligned}
$$

In addition, since

$$
\begin{aligned}
& -\bar{\gamma} L y^{p+1}+[\bar{\omega}(2-\bar{\omega}) M+\bar{\gamma} L] y^{p}+\bar{\omega}(2-\bar{\omega}) q \\
& =-\bar{\gamma} L y^{p+1}-+[\bar{\omega}(2-\bar{\omega}) M+\bar{\gamma} L]+\bar{\omega}(2-\bar{\omega}) q \leq-\gamma L\left(y^{p+1}-y^{p}\right)+\omega(2-\omega)\left(M y^{p}+q\right) .
\end{aligned}
$$

Therefore

$$
\begin{aligned}
y^{p+1} \geq & \left(y^{p}-D^{-1}\left[-\gamma L\left(y^{p+1}-y^{p}\right)\right.\right. \\
& \left.\left.+\omega(2-\omega)\left(M y^{p}+q\right)\right]\right)_{+}=f\left(y^{p}\right) .
\end{aligned}
$$

We verify (11) by induction. In fact, when $\mathrm{k}=0$, the inequality (12) is trivial. Suppose that (11) holds for some positive integer p. $z^{k} \leq y^{k}, k=0,1,2, \ldots p$. Then by Theorem 4.1 and the inequality (12), we get $z^{p+1}=f\left(z^{p}\right) \leq f\left(y^{p}\right) \leq y^{p+1}$ and hence $z^{k} \leq y^{k}, k=0,1,2, \ldots$. This completes the proof.

Remark. Theorem 4.2 shows that suitable increase of the parameter $\omega$ can accelerate the convergence rate of the SAOR method. Furthermore, the parameter $\gamma=\omega=1$ can result in the fastest convergence rate of the SAOR method under the restrictions made in Theorem 4.2 and it implies that the optimal parameter in general should be $\omega, \gamma \in[1, \infty)$.

\section{Numerical Results}

In this section, we present numerical results to show the efficiency of SAOR method and AOR method for different cases. The codes are written by Matlab.

Example. We consider the LCP(M,q) with

$$
M=\left(\begin{array}{ccccccc}
S & -I & -I & O & \cdots & O & O \\
O & S & -I & -I & \cdots & O & O \\
O & O & S & -I & \cdots & O & O \\
\vdots & \vdots & \ddots & \ddots & \ddots & \ddots & \vdots \\
\vdots & \vdots & \ddots & \ddots & \ddots & \ddots & -I \\
\vdots & \vdots & & \ddots & \ddots & S & -I \\
O & O & \cdots & \cdots & O & O & S
\end{array}\right) \in R^{n \times n}, q \in\left(\begin{array}{c}
-1 \\
1 \\
-1 \\
\vdots \\
(-1)^{n-1} \\
(-1)^{n}
\end{array}\right) \in R^{n}
$$


where $S=\operatorname{tridiag}(1,8,-1) \in R^{\bar{n} \times \bar{n}}, I \in R^{\bar{n} \times \bar{n}}$ is the identity matrix, and $\bar{n}^{2}=n$ and take $n=900$ for example. It is known that $\mathrm{M}$ is a strictly diagonally dominant matrix and thus is an H-matrix. So the $\mathrm{LCP}(\mathrm{M}, \mathrm{q})$ has an unique solution $z^{*} \in R^{n}$.

For the test problem, we take the initial vector $z^{0}=(5,5, \cdots, 5)^{T}$. The termination criterion for the iterative methods is $\delta\left(z^{k}\right)=\left\|z^{k}-z^{k-1}\right\|_{\infty}<10^{-6}$, and let NI and CT denote the number of iterations and CPU time, respectively.

Table 1. The number of iterations and cpu time of Saor method and Aor method with different $\omega$ and $\gamma$

\begin{tabular}{lccccc}
\hline \multirow{2}{*}{$\omega$} & $\gamma$ & \multicolumn{2}{c}{ SAOR method } & \multicolumn{2}{c}{ AOR method } \\
\cline { 3 - 6 }$\omega=$ & $\gamma=0.01$ & 1.508788 & 94 & 15.953723 & 186 \\
0.02 & $\gamma=0.02$ & 1.510670 & 94 & 15.788688 & 186 \\
$\omega=$ & $\gamma=0.1$ & 0.173260 & 11 & 1.635054 & 19 \\
0.2 & $\gamma=0.2$ & 0.183620 & 11 & 1.615800 & 19 \\
$\omega=$ & $\gamma=0.5$ & 0.048242 & 3 & 0.261636 & 3 \\
1.0 & $\gamma=1.0$ & 0.047447 & 3 & 0.255026 & 3 \\
$\omega=$ & $\gamma=1.1$ & 0.048009 & 3 & 0.253369 & 3 \\
1.2 & $\gamma=1.2$ & 0.048526 & 3 & 0.254806 & 3 \\
$\omega=$ & $\gamma=1.4$ & 0.080753 & 5 & 0.252666 & 3 \\
1.5 & $\gamma=1.5$ & 0.078928 & 5 & 0.256678 & 3 \\
$\omega=$ & $\gamma=1.8$ & 0.299969 & 19 & 0.254958 & 3 \\
1.9 & $\gamma=1.9$ & 0.303658 & 19 & 0.253611 & 3 \\
\hline
\end{tabular}

In above Table, we report the NI and CT of SAOR and AOR methods with different parameters of $\omega$ and $\gamma$. We can easily find that SAOR method are more efficient than AOR method when $0<\gamma \leq \omega \leq 1$; on the contrary while when $1<\gamma \leq \omega<2$, AOR method is more efficient than SAOR method. Furthermore, it can be observed that the method is the most efficient when $\omega=\gamma=1$ than any other value when $0<\gamma \leq \omega \leq 2$. Our obtained numerical results confirm Theorem 4.2 remarkably.

\section{References}

[1] A. Berman and R. J. Plemmons, Nonnegative Matrices in the Mathematical Sciences, 3rd ed., SIAM, Philadelphia, 1994.

[2] R. W. Cottle, G. H. Golub and R. S. Sacher, On the solution of large structured linear complementarity problems, Technical Report STAN-CS-74 439, Stanford University, Stanford, CA, 1974.

[3] M. D. Koulisianis and T. S. Papatheodorou, "Improving projected successive overrelaxation method for linear complementarity problems," Appl. Numer. Math, vol. 45, no. 1, pp. 29-40, 2003.

[4] D. Yuan and Y. Song, "Modified AOR Methods for linear complementarity problem", Appl. Math. Comput, vol. 140, no. 1, pp. 53-67, 2003.

[5] Y. Li and P. Dai, "Generalized AOR methods for linear complementarity problem," Appl. Math. Comput, vol. 188, no.1, pp. 7-18, 2007.

[6] Z. Z. Bai and D. J. Evans, "Matrix multisplitting relaxation methods for the linear complementarity problem,” Int. J. Comput. Math, vol. 63, no. 3-4, pp. 309-326, 1997. 
[7] Z. Z. Bai, "On the convergence of the multisplitting methods for the linear complementarity problem", SIAM J. Matrix Anal. Appl, vol. 21, no. 1, pp. 67-78, 1999.

[8] M. Wu, L. Wang and Y. Song, "Preconditioned AOR iterative method for linear systems", Appl. Numer. Math, vol. 57, no. 5-7, pp. 672-685, 2007.

[9] R. S. Varga, Matrix iterative Analysis, Springer-Verlag, New York, 2000.

[10] O. L. Mangasarian, "Solution of symmetric linear complementarity problems by iterative methods," $J$. Optim. Theory Appl, vol. 22, no.4, pp. 465-485, 1977.

[11] B. H. Ahn, "Solution of nonsymmetric linear complementarity problems by iterative methods", J. Optim. Theory Appl, vol. 33, no. 2, pp. 175-185, 1981. 